\title{
Modified Luneburg Lens Based on Metamaterials
}

\author{
Haibing Chen, Qiang Cheng, Aihua Huang, Junyan Dai, Huiying Lu, \\ Jie Zhao, Huifeng Ma, Weixiang Jiang, and Tiejun Cui
}

State Key Laboratory of Millimeter Waves, Department of Radio Engineering, Southeast University, Nanjing 210096, China

Correspondence should be addressed to Haibing Chen; chhbyxyy@126.com

Received 7 October 2014; Revised 22 January 2015; Accepted 29 January 2015

Academic Editor: Giuseppe Castaldi

Copyright (C) 2015 Haibing Chen et al. This is an open access article distributed under the Creative Commons Attribution License, which permits unrestricted use, distribution, and reproduction in any medium, provided the original work is properly cited.

\begin{abstract}
We present the design, fabrication, and experimental characterization of a modified two-dimensional Luneburg lens based on bulk metamaterials. The lens is composed by a number of concentric layers. By varying the geometric dimensions of unit cells in each layer, the gradient refractive index profile required for the modified Luneburg lens can be achieved. The cylindrical waves generated from a point source at the focus point of the lens could be transformed into plane waves as desired in the microwave frequency. The proposed modified Luneburg lens can realize wide-angle beam scanning when the source moves along the circumferential direction inside the lens. Numerical and experimental results validate the performance of the modified Luneberg lens.
\end{abstract}

\section{Introduction}

In the past a few years, the emergence of metamaterials have aroused great interests due to their fascinating properties and potential applications in microwave and optical engineering, like the superlens, invisible cloak, and other transformation devices [1-3]. Metamaterials are usually composed of periodic resonant or nonresonant structures, and the effective properties can be inherited from the design variations of the unit cells. Such artificial materials provide us a powerful tool for manipulation of electromagnetic waves on the subwavelength scale, which makes the capture and control of electromagnetic near fields in designed structures become possible.

In particular, the gradient refractive index (GRIN) metamaterials play important roles in the design of new concept devices, whose index profile can be varied gradually according to the designs. By arranging the same type of metamaterial element with different geometries, the spatial gradient of the refraction index can be finally introduced. The GRIN metamaterials can provide larger refraction index contrast than the natural materials, and they can also be tuned by electric or optical means, which makes them more attractive in practical applications.
One typical application of GRIN metamaterials is the microwave lens, which can transform the cylindrical or spherical waves into planar waves. Compared to the traditional dielectric lens, the GRIN metamaterial lens offers more freedom for designers to elaborate their work as stated above. Up to now, a number of GRIN metamaterial lenses have been demonstrated, such as Maxwell fisheye lenses, Eaton lenses, Wood lenses, and Luneburg lenses [3-8]. Owing to the compact size, reduced weight, and convenience of fabrication, the GRIN metamaterial lens has a number of advantages compared to the traditional lens [9-14].

In this paper, we have designed and fabricated a modified 2D-Luneburg lens based on the GRIN metamaterials [1517]. Different from the traditional Luneburg lens, the current lens can be excited internally, making it more flexible in practical applications [18]. The modified Luneburg lens can realize wide-angle scanning when the excitation probe moves along the circumferential direction inside the lens. By periodically placing I-shaped units with varied dimensions in the radial direction, we can get the desired refractive index profile. A probe antenna has been inserted within the sample as the excitation. The near-field electric distributions have been measured and plotted. Excellent plane waves could be observed outside the modified Luneburg lens, which shows good performance of the designed sample. 


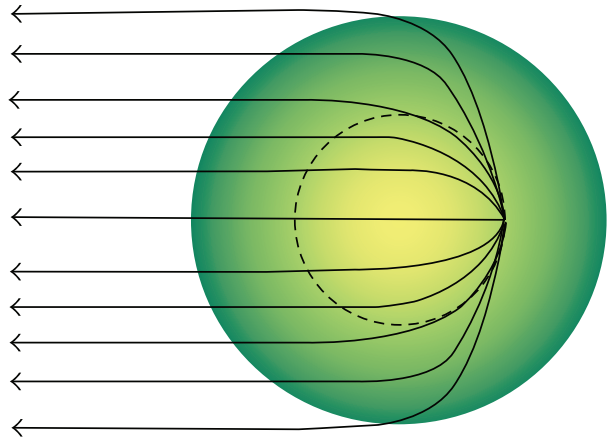

(a)
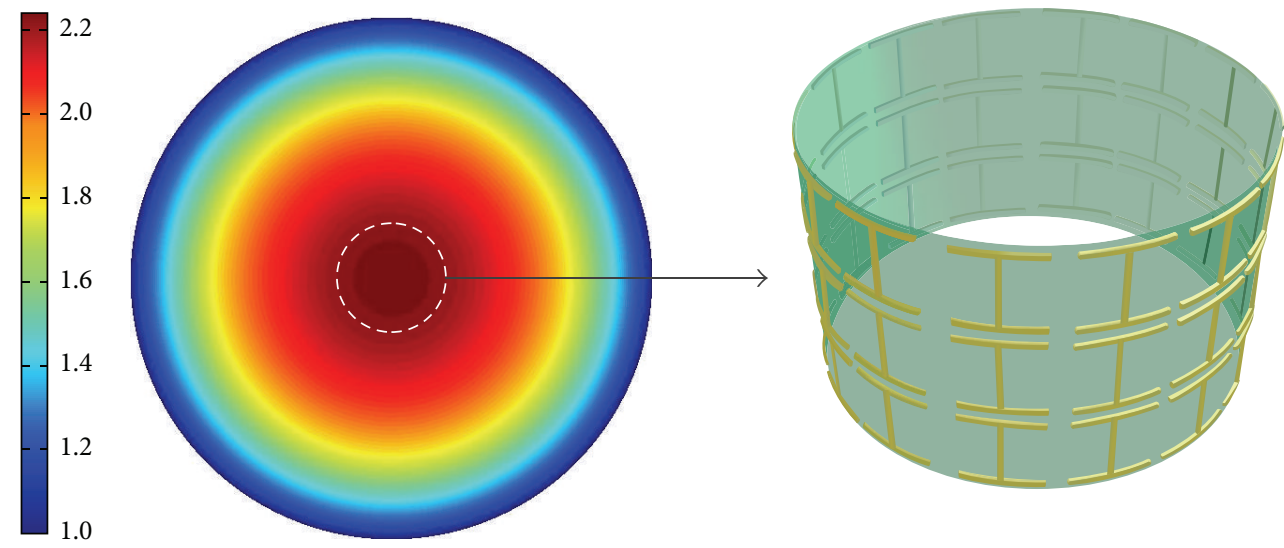

(b)

FIGURE 1: (a) Ray trajectories through the modified Luneburg lens, where the source is located inside the lens. (b) Refraction index profile for the modified Luneburg lens; the inset shows one of the concentric layers of the designed lens.

\section{Design and Simulation}

For a two-dimensional modified Luneburg lens as shown in Figure 1, the radially inhomogeneous refraction index profile satisfies

$$
n(r)=\frac{\left(a^{2}+f^{2}-r^{2}\right)^{1 / 2}}{f} \quad(0 \leq r \leq a),
$$

where $a$ is the radius of the lens and $f$ is the distance from the lens center to the focus. The medium outside the lens is free space with the relative refraction index $n(a)=1$, in order to keep impedance match at the lens boundary. Figure 1(a) shows the ray trajectories through the modified Luneburg lens. The rays from the internal source are transmitted through the lens to produce a parallel beam on the other side of the lens. Here we choose $f<a$ to ensure that the focus is located inside the lens. When $f=a / 2$, the refraction index profile inside the lens can be found in Figure 1(b), ranging from 1 to 2.236 .

The unit cell of the modified Luneburg lens is shown in Figure 2, which is actually a nonresonant I-shaped metallic structure upon a dielectric substrate [19]. Electric response could be exhibited for the I-shaped unit under the illumination of external electric field, resulting in the variation of the effective permittivity according to effective medium theory [20]. Here, the reason we choose I-shaped structure as our unit cell is that it has few parameters to tune. Actually, other nonresonant structures having electric response can be our unit cell under rational design. In our design, the two horizontal bars are kept to have the same length to the vertical bar in each unit. Therefore it is quite easy to achieve different permittivity distributions as required by simply tuning the length of the bar. Due to the periodicity of the composing unit, it is hard to realize a modified Luneburg lens with continuous refraction index as required by (1). To make an approximation, the discretization method can be utilized to realize the desired modified Luneburg lens. Here we divide the whole lens into fifteen concentric layers. All the units in the same layer have the equal geometric dimensions, as shown in Figure 1(b). FR4 is chosen as the substrate with the relative permittivity 4.3 and the thickness $0.2 \mathrm{~mm}$. The periodicity of each unit is set to be $3.333 \mathrm{~mm}$. All the geometrical dimensions of the I-shaped unit have been shown in Figure 2(a) except the length of the vertical bar at different layers, which should be carefully optimized to meet the requirement of the refraction index profile at $10 \mathrm{GHz}$.

In order to show the relationship between the geometry dimensions and the constitutive parameters of the I-shaped unit, numerical simulations have been made to get the corresponding refraction index at $f=10 \mathrm{GHz}$ following the standard retrieval procedure [21]. The length $w$ in Figure 2 is chosen to change gradually to tune the refraction index as 


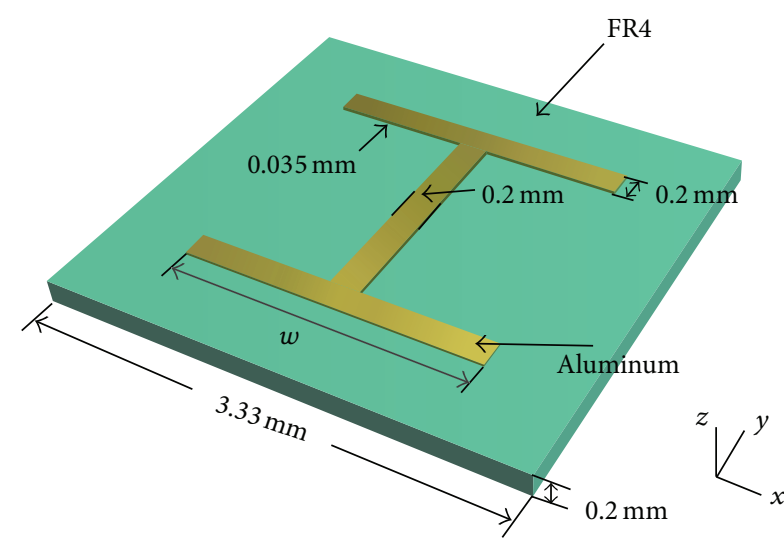

(a)

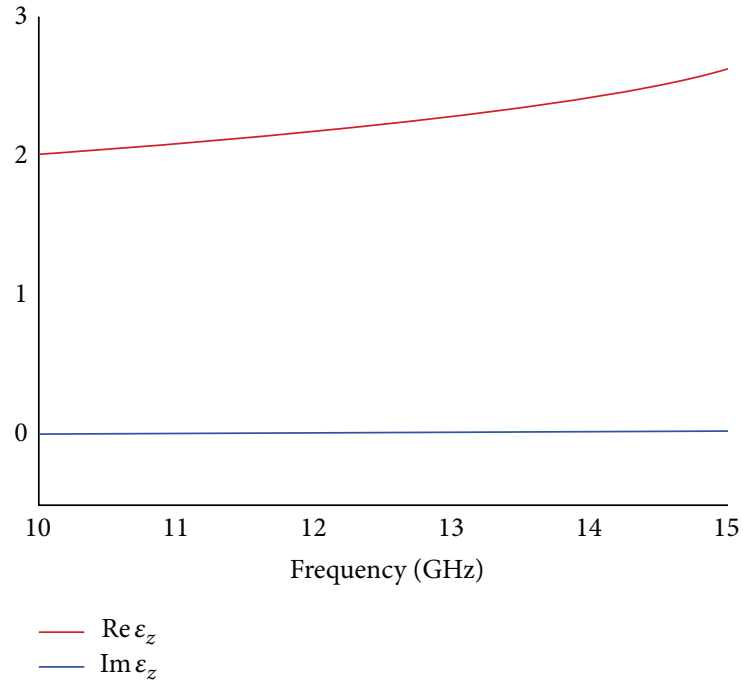

(b)

FIgURE 2: The unit cell of the modified Luneburg lens. (a) The perspective view. (b) The effective $\varepsilon_{z}$ of the unit.

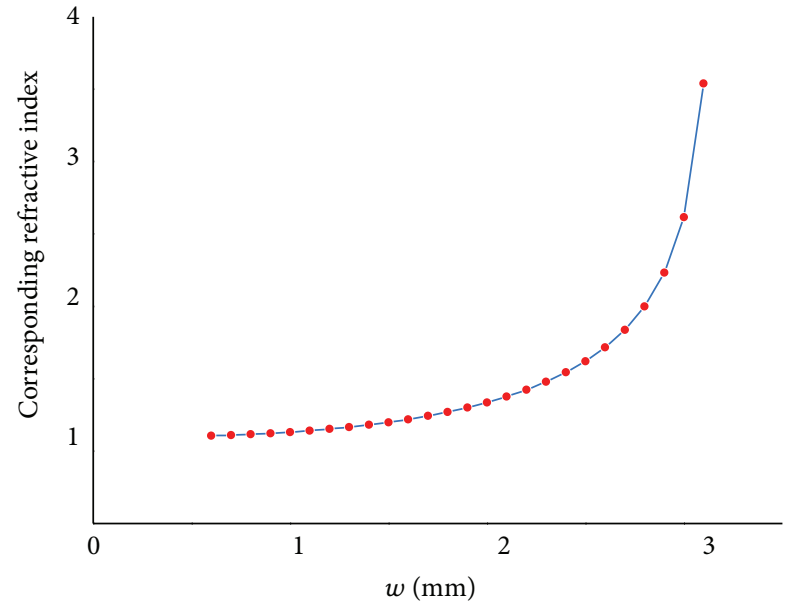

FIGURE 3: The relationship between the parameter $w$ (shown in Figure 2(a)) and the corresponding effective refractive index.

required by the modified Luneburg lens. The width of the bars is fixed to be $0.2 \mathrm{~mm}$. From Figure 3, the effective refraction index increases gradually with the growth of $w$, ranging from 1 to 3.5 .

Like the traditional 2D Luneberg lenses, the modified one can also realize beam shaping and beam scanning, which can transform cylindrical waves into plane waves in various directions. To demonstrate the performance of the proposed lens, full wave numerical simulations have been made by using the software package COMSOL. A 2D point source has been inserted into the lens as the excitation, as depicted by the cross in Figure 4. The radius of the lens is set to be $50 \mathrm{~mm}$. The electric field inside and outside the lens has been illustrated in Figure 4, where the lens with continuous and discrete refraction index profile has been taken into considerations. By comparing Figures 4(a) and 4(b), it is clear that unavoidable scattering has emerged due to the discretization in the practical realization for its impedance mismatch. However, the beam shaping phenomenon is still quite obvious. Under ideal conditions, there should be almost no energy loss when electromagnetic waves run through the lens, since the imaginary part of the effective dielectric constant is approximately zero as shown in Figure 2(b). However, loss indeed exists because of the productive deviation and tiny impedance mismatch to the air part. By moving the source along the circumference, it is possible to realize beam scanning toward different directions, as shown in Figure 4(c), which is critically important for radar detections.

\section{Fabrication and Measurement}

To verify the performance of the designed Luneburg lens, we have fabricated the sample (see Figure 5), which is constituted by 15 concentric layers with the gap between adjacent layers $3.333 \mathrm{~mm}$. The I-shaped unit is etched on the copper cladding of the FR4 substrate. All the layers have been inserted into a Styrofoam board to keep the sample stable in the experiment. Each layer has 3 units in the vertical direction with the height $10 \mathrm{~mm}$, as required by the scanning waveguide mentioned later.

A two-dimensional microwave scanning apparatus has been used in the experiment to obtain the near field distributions of the fabricated sample, which is actually a planar waveguide whose dominant mode is TEM mode [20]. Two motors are placed below the bottom metallic plate, which can scan the area of $200 \mathrm{~mm} * 200 \mathrm{~mm}$ under the control of the computer. Both the excitation and detection probes have been connected to the vector network analyzer (Agilent N5230C), and the measured data will be sent back to the computer for recording and processing. In this experiment, the electric field within a $2 \mathrm{D}$ plane ( $1 \mathrm{~mm}$ higher above the sample) has been measured with the scanning of detection probe. The gap 


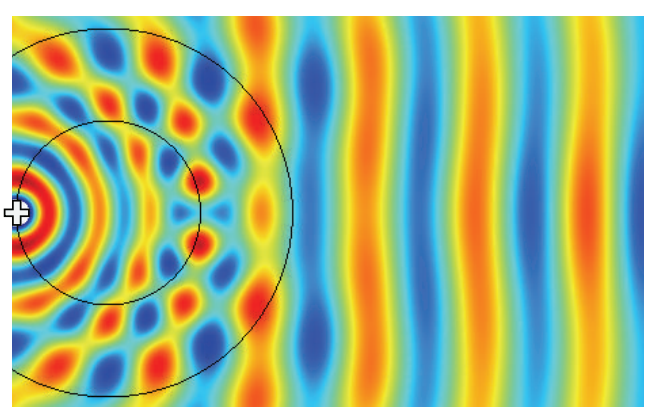

(a)

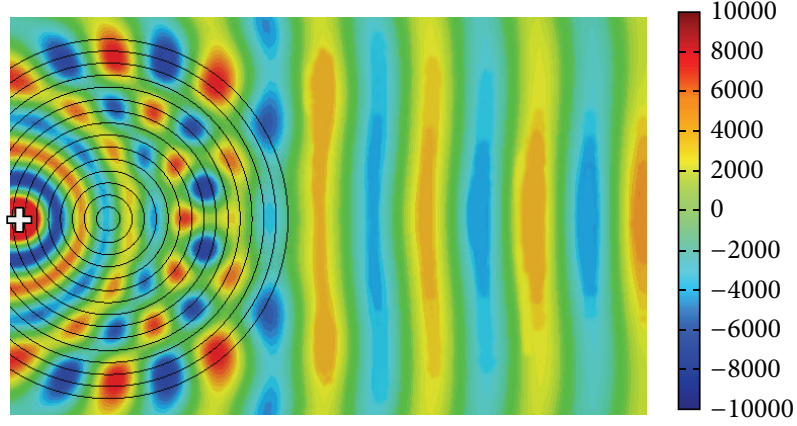

(b)

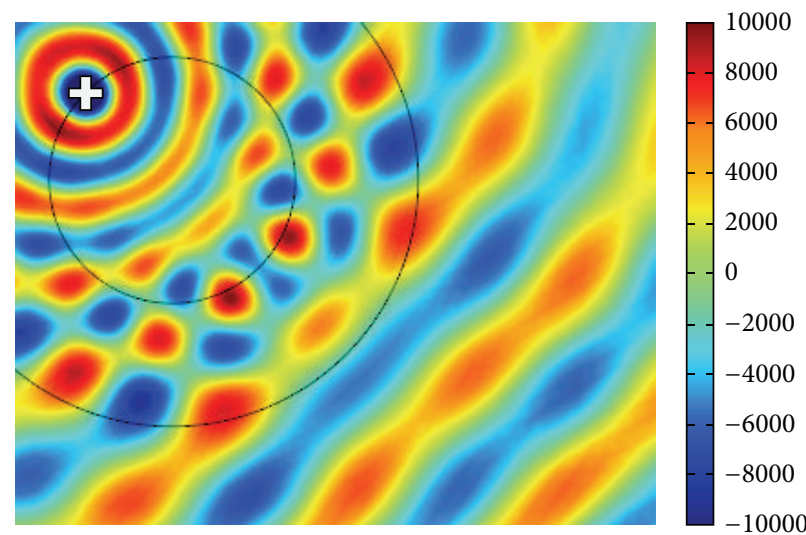

(c)

Figure 4: Simulated electric field distributions for a point source embedded within the modified Luneburg lens at $10 \mathrm{GHz}$. (a) The lens has the ideal refraction index distributions as shown in (1). (b) The refractive index distributions of the lens are discretized. (c) Beam scanning for a point source moving along the circumferential direction.

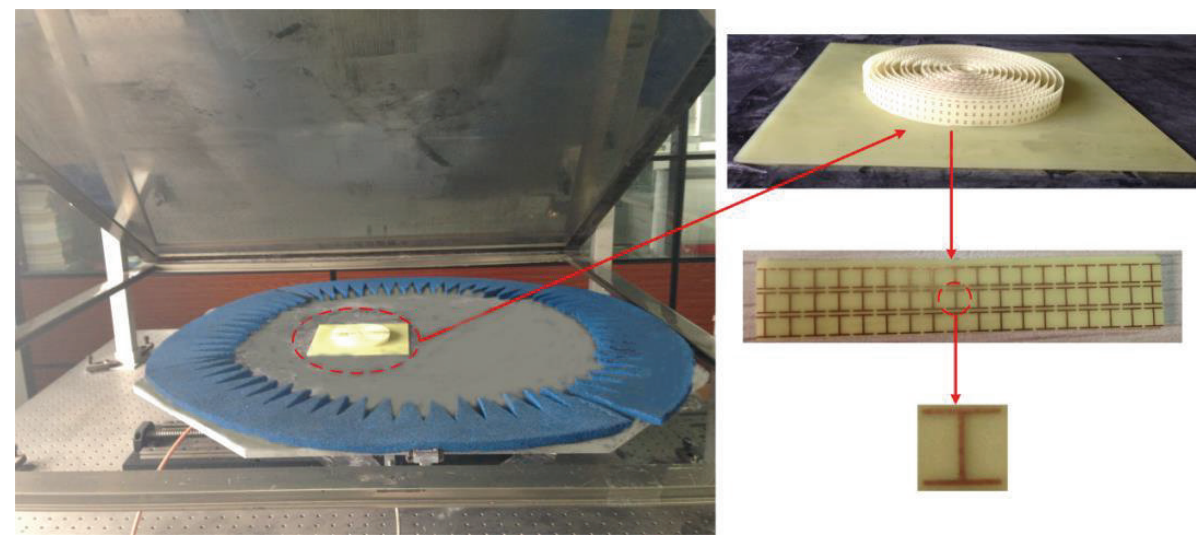

FIgURE 5: The experimental setup of the modified Luneburg lens, where the inset shows the details of the sample.

between the bottom and the upper plate is $12 \mathrm{~mm}$ to avoid the emergence of higher order modes for the planar waveguide. The excitation probe lies between the seventh and eighth layer of the sample to generate the cylindrical waves. Microwave absorption materials are placed at the edge of bottom plate to reduce the unwanted wave reflections.

The electric field distribution around the sample is measured and plotted, as shown in Figures 6(a) and 6(b), where the location of the sample is marked by the red dashed lines.
It can be observed that cylindrical waves propagate on the left side of the sample and plane waves propagate on the right side of the sample. We choose $10.5 \mathrm{GHz}$ as our observed frequency point rather than the designed frequency point $10 \mathrm{GHz}$, because it has frequency deviation owing to the fabrication error. There is also tiny impedance mismatch at the boundary of the fabricated sample, since the effective refractive index for the outmost layer is 1.141, which is slightly higher than that of air, leading to the unwanted scattering. 


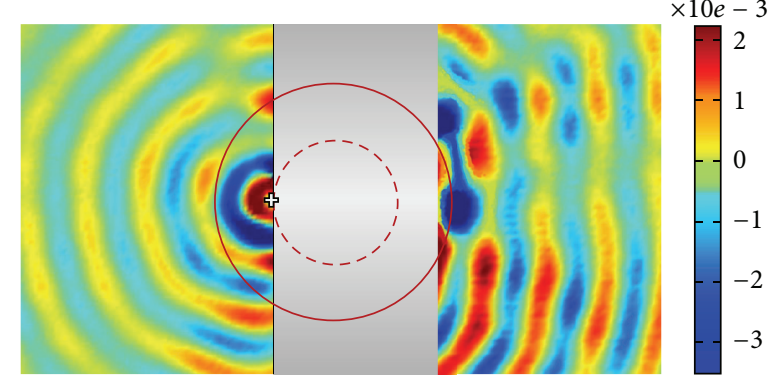

(a)

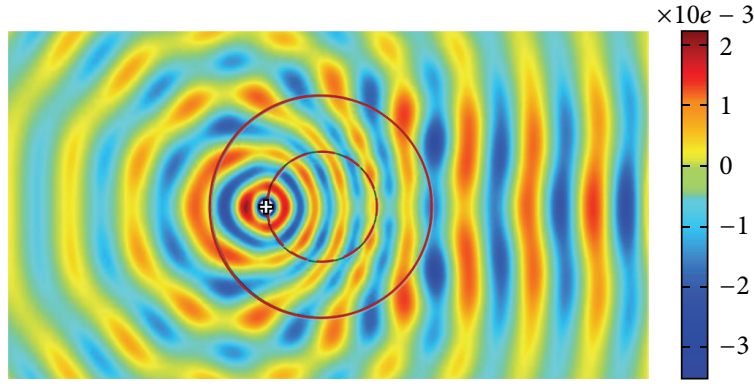

(b)

FIGURE 6: Electric field distributions for a point source embedded within the fabricated lens at the frequency of $10.5 \mathrm{GHz}$, which is indicated by the cross. (a) Measured. (b) Simulated.

The grey region represents the region where we cannot plot due to the limitation of the testing platform. Comparing the simulation result with the measurement result at the frequency of $10.5 \mathrm{GHz}$, we can find that they agree well, showing great potentials for microwave and optical applications in the future.

\section{Conclusion}

In this paper, a modified 2D-Luneberg lens has been designed, fabricated, and experimentally characterized based on the I-shaped units. Gradient index distributions have been realized by adjusting the dimensions of the basic unit based on the effective medium theory. Experimental results show that cylindrical waves can be transformed into plane waves, which is consistent with the theoretical predictions.

\section{Conflict of Interests}

The authors declare that there is no conflict of interests regarding the publication of this paper.

\section{Acknowledgments}

This work was supported by National Science Foundation of China (61138001 and 61171026), National High Tech (863) Projects (2011AA010202 and 2012AA030402), 111 Project (111-2-05), and the Natural Science Foundation of Jiangsu Province (BK2012019).

\section{References}

[1] J. B. Pendry, D. Schurig, and D. R. Smith, "Controlling electromagnetic fields," Science, vol. 312, no. 5781, pp. 1780-1782, 2006.

[2] D. Schurig, J. J. Mock, B. J. Justice et al., "Metamaterial electromagnetic cloak at microwave frequencies," Science, vol. 314, no. 5801, pp. 977-980, 2006.

[3] U. Leonhardt, "Optical conformal mapping," Science, vol. 312, no. 5781, pp. 1777-1780, 2006.

[4] T. Zentgraf, Y. M. Liu, M. H. Mikkelsen, J. Valentine, and X. Zhang, "Plasmonic Luneburg and Eaton lenses," Nature Nanotechnology, vol. 6, no. 3, pp. 151-155, 2011.
[5] A. Di Falco, S. C. Kehr, and U. Leonhardt, "Luneburg lens in silicon photonics," Optics Express, vol. 19, no. 6, pp. 5156-5157, 2011.

[6] X. D. Wu and J.-J. Laurin, "Fan-beam millimeter-wave antenna design based on the cylindrical Luneberg lens," IEEE Transactions on Antennas and Propagation, vol. 55, no. 8, pp. 2147-2156, 2007.

[7] L. H. Gabrielli and M. Lipson, "Integrated Luneburg lens viaultra-strong index gradient on silicon," Optics Express, vol. 19, no. 21, pp. 20122-20127, 2011.

[8] J. Hunt, T. Tyler, S. Dhar et al., "Planar, flattened Luneburg lens at infrared wavelengths," Optics Express, vol. 20, no. 2, pp. 17061713, 2012.

[9] W. X. Jiang, H. F. Ma, Q. Cheng, and T. J. Cui, "Virtual conversion from metal object to dielectric object using metamaterials," Optics Express, vol. 18, no. 11, pp. 11276-11281, 2010.

[10] A. Dhouibi, S. N. Burokur, A. de Lustrac, and A. Priou, "X-band metamaterial-based Luneburg lens antenna," in Proceedings of the IEEE Antennas and Propagation Society International Symposium (APSURSI '13), pp. 1292-1293, IEEE, July 2013.

[11] Q. Cheng, H. F. Ma, and T. J. Cui, "Broadband planar Luneburg lens based on complementary metamaterials," Applied Physics Letters, vol. 95, no. 18, Article ID 181901, 2009.

[12] J. B. Pendry, "Negative refraction makes a perfect lens," Physical Review Letters, vol. 85, no. 18, pp. 3966-3969, 2000.

[13] H. F. Ma, X. Chen, H. S. Xu, X. M. Yang, W. X. Jiang, and T. J. Cui, "Experiments on high-performance beam-scanning antennas made of gradient-index metamaterials," Applied Physics Letters, vol. 95, no. 9, Article ID 094107, 2009.

[14] H. F. Ma and T. J. Cui, "Three-dimensional broadband and broad-angle transformation-optics lens," Nature Communications, vol. 1, no. 8, article 124, 2010.

[15] T. Koschny, M. Kafesaki, E. N. Economou, and C. M. Soukoulis, "Effective medium theory of left-handed materials," Physical Review Letters, vol. 93, no. 10, Article ID 107402, 2004.

[16] Y. Wu, Y. Lai, and Z.-Q. Zhang, "Effective medium theory for elastic metamaterials in two dimensions," Physical Review BCondensed Matter and Materials Physics, vol. 76, no. 20, Article ID 205313, 2007.

[17] D. Headland, W. Withayachumnankul, M. Webb, and D. Abbott, "Beam deflection lens at terahertz frequencies using a hole lattice metamaterial," in Proceedings of the 38th International Conference on Infrared, Millimeter, and Terahertz Waves (IRMMW-THz '13), vol. 10, pp. 1-2, IEEE, Mainz, Germany, September 2013. 
[18] A. S. Gutman, "Modified Luneberg lens," Journal of Applied Physics, vol. 25, no. 7, pp. 855-859, 1954.

[19] M. Choi, S. H. Lee, Y. Kim et al., "A terahertz metamaterial with unnaturally high refractive index," Nature, vol. 470, no. 7334, pp. 369-373, 2011.

[20] W. X. Jiang, T. J. Cui, G. X. Yu, X. Q. Lin, Q. Cheng, and J. Y. Chin, "Arbitrarily elliptical-cylindrical invisible cloaking," Journal of Physics D: Applied Physics, vol. 41, no. 8, Article ID 085504, 2008.

[21] D. R. Smith, D. C. Vier, T. Koschny, and C. M. Soukoulis, "Electromagnetic parameter retrieval from inhomogeneous metamaterials," Physical Review E-Statistical, Nonlinear, and Soft Matter Physics, vol. 71, no. 3, Article ID 036617, 2005. 

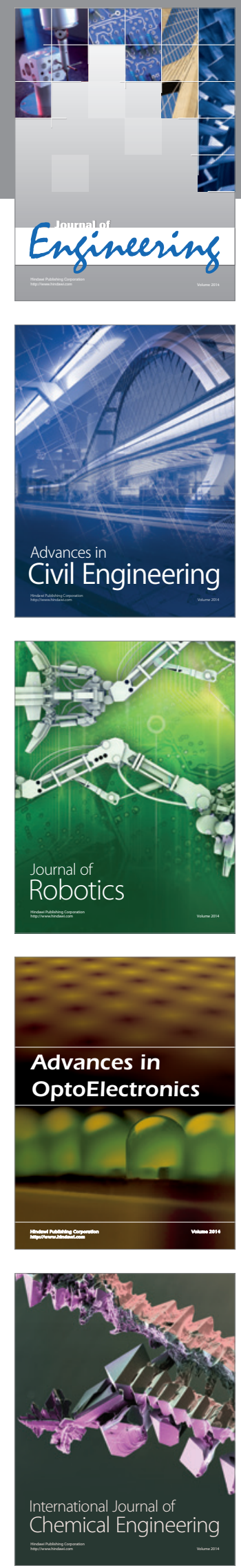

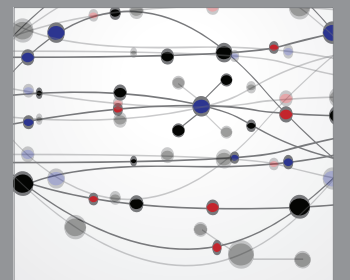

The Scientific World Journal
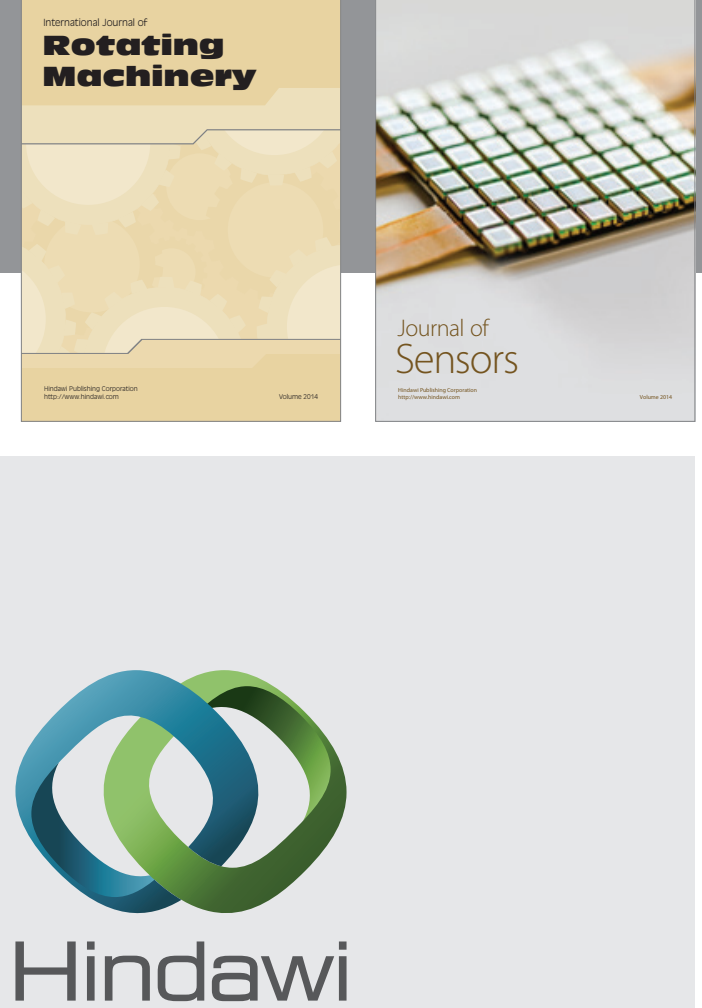

Submit your manuscripts at http://www.hindawi.com
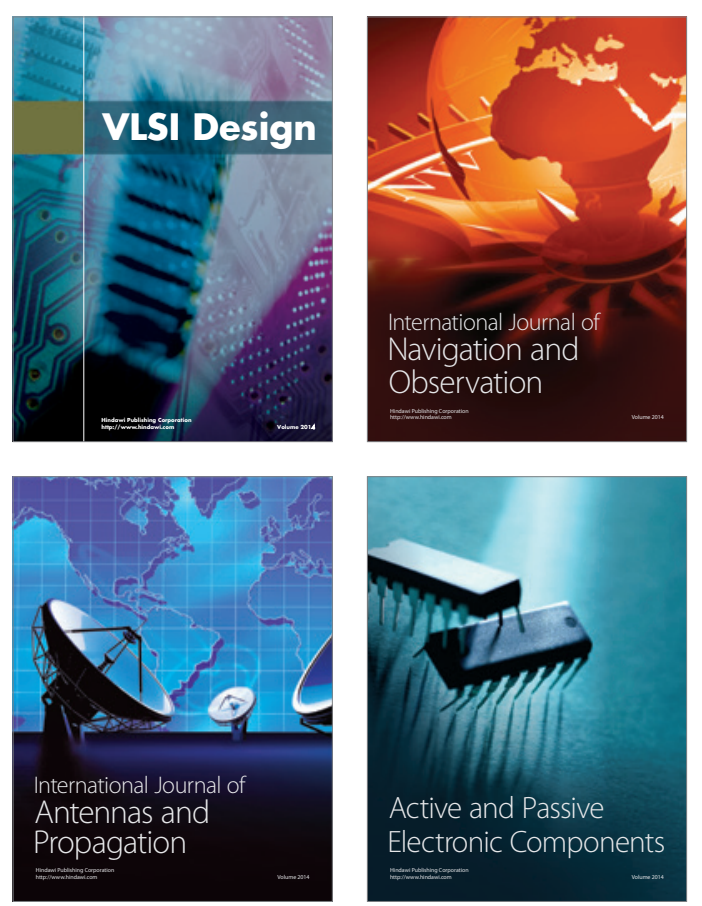
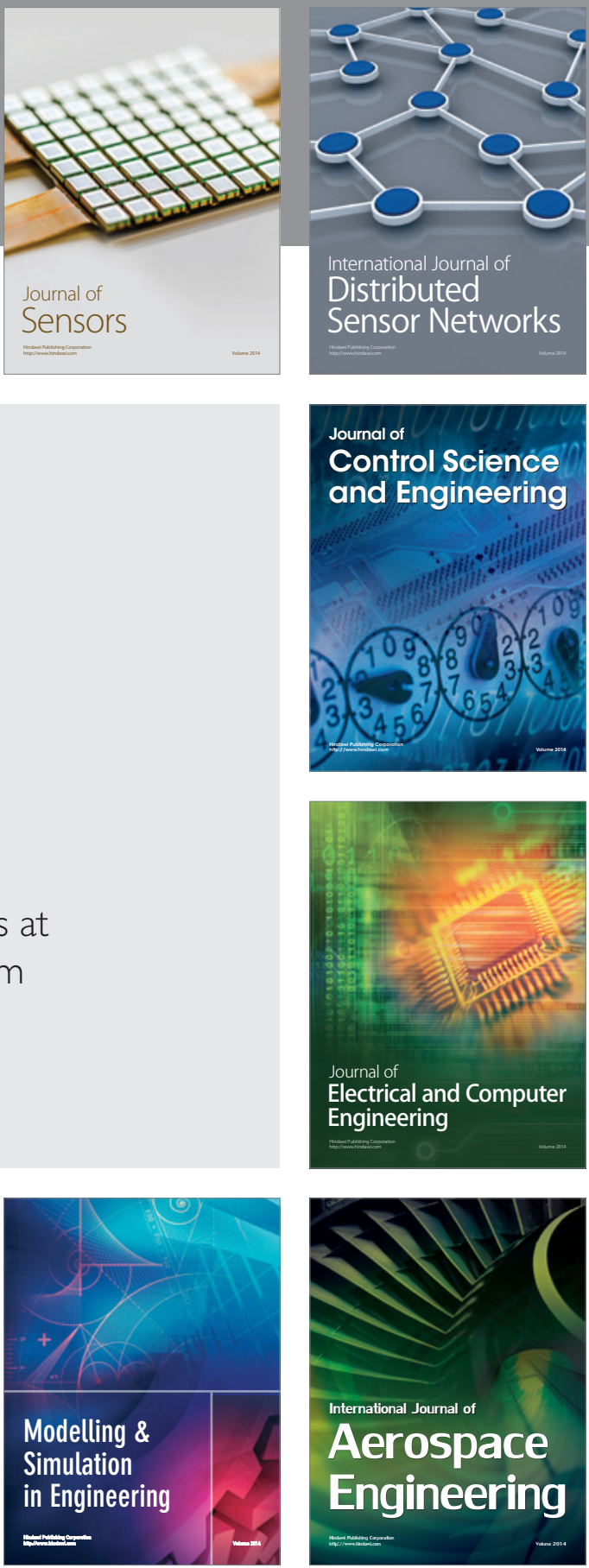

Journal of

Control Science

and Engineering
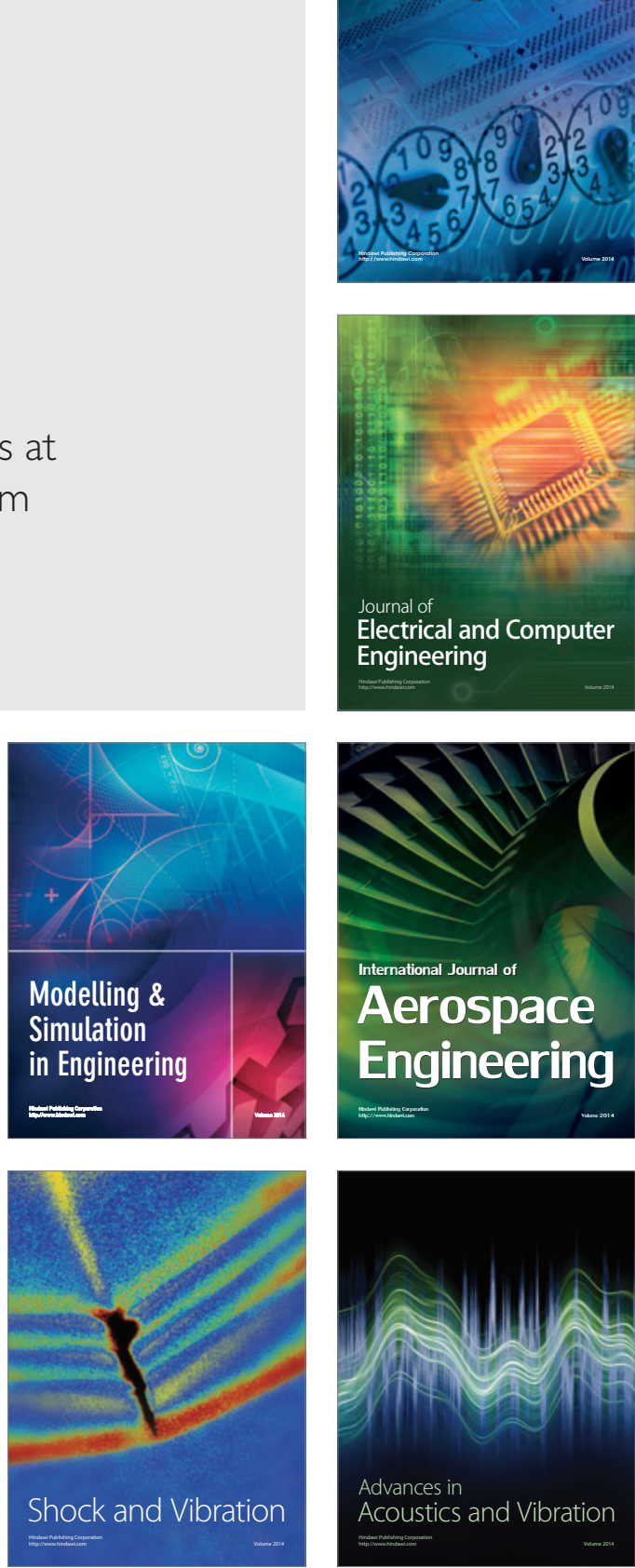